J. Austral. Math. Soc. 23 (Series A) (1977), 28-41.

\title{
ENLARGING THE MUNN REPRESENTATION OF INVERSE SEMIGROUPS
}

\author{
N. R. REILLY
}

(Received 10 September, 1975)

\begin{abstract}
The inverse semigroup $T_{E}$ of isomorphisms of principal ideals of $E$ onto principal ideals of $E$, where $E$ is a semilattice, has been introduced and studied by Munn $(1966,1970)$. He showed that, for any inverse semigroup $S$ with semilattice $E$, there is a representation of $S$ by an inverse subsemigroup of $T_{E}$. The Munn representation, however, is not always faithful. In this paper, the possibility is considered of enlarging the carrier set $E$ of the Munn representation in order to obtain a faithful representation of $S$ as an inverse subsemigroup of a structure resembling $T_{E}$ in many ways. A structure $X$ is obtained by replacing each element of $E$ by a set. Then $X=$ $\cup\left\{X_{e}: e \in E\right\}$, where $X_{e}$ denotes some set, has a natural pre-order relation $\leqslant$ (where $x \leqslant y$ if and only if $x \in X_{e}, y \in X$, and $\left.e \leqq f\right)$ inherited from $E$ such that if $\tau=\{(x, y) \in X \times X ; x \leqslant y$ and $y \leqslant x\}$ then $X / \tau$ is isomorphic to $E$. Such a set $X$ is referred to as a pre-semilattice with semilattice $E$. If $T_{X}$ denotes the set of all isomorphisms of principal ideals of $X$ onto principal ideals of $X$ then $T_{X}$ is an inverse semigroup. Basic properties of $T_{X}$ are considered. It is shown that when $X$ is locally uniform, that is, when $\left|X_{e}\right|=\left|X_{f}\right|$, for all $e, f \in E, T_{X}$ may be described as a wreath product of a permutation group with $T_{\mathrm{E}}$.

The set $S$ itself is a presemilattice with semilattice $E$ with respect to the pre-order $\leqslant$ defined by $a \leqslant b$ if and only if $a^{-1} a \leqq b^{-1} b$. It is then shown that the Vagner-Preston representation embeds $S$ as a full inverse subsemigroup of $T_{s}$. As an application of these concepts the following result is established. Let $R$ and $S$ be inverse semigroups and let $\theta_{1}\left(\theta_{2}\right)$ be an isomorphism of a semilattice $E$ onto the semilattice of $R(S)$. Then there exists a locally uniform presemilattice $W$ and embeddings $\varphi_{1}, \varphi_{2}$ of $R$ and $S$, respectively, as full inverse subsemigroups of $T_{w}$ such that (1) $\theta_{1} \varphi_{1}=\theta_{2} \varphi_{2}$ and (2) $\left(e \theta_{1} \varphi_{1}, e \theta_{2} \varphi_{2}\right) \in \mathscr{D}$ if and only if $E e$ is isomorphic to $E f$.
\end{abstract}

\section{Introduction}

For a semilattice $E$, Munn (1966) introduced the inverse semigroup $T_{E}$ of all isomorphisms of principal ideals of $E$ onto principal ideals of $E$. He then showed that, for any inverse semigroup $S$ with semilattice $E$, there is a natural representation of $S$ by an inverse subsemigroup of $T_{E}$. This representaion, which we shall refer to as the Munn representation, is not always faithful and, in fact, induces the maximum idempotent separating congruence on $S$. Here 
the possibility is considered of enlarging the carrier set $E$ of the Munn representation in order to obtain a faithful representation of $S$ as an inverse subsemigroup of a structure resembling $T_{E}$. Intuitively this structure is obtained by replacing each element of $E$ by a set. This enlargement $X=$ $\cup\left\{X_{e}: e \in E\right\}$, say, of $E$ has a natural pre-order relation $\leqslant$ (where $x \leqslant y$ if and only if $x \in X_{e}, y \in X_{f}$ and $\left.e \leqq f\right)$ inherited from $E$ such that if $\tau=$ $\{(x, y): x \leqslant y$ and $y \leqslant x\}$ then $X / \tau$ is isomorphic to $E$. We call $X$ a presemilattice with semilattice $E$. If $T_{X}$ denotes the set of all isomorphisms of principal ideals of $X$ onto principal ideals of $X$ then $T_{X}$ is an inverse semigroup. The first section is devoted to basic properties of $T_{X}$. In the second section we consider the case where $X$ is locally uniform, that is, where $\left|X_{e}\right|=$ $\left|X_{f}\right|$, for all $e, f \in E$. For such a locally uniform $X$, it is shown that $T_{X}$ may be described as a wreath product of a permutation group with $T_{E}$.

It is then observed that any inverse semigroup $S$ is a presemilattice with respect to the pre-order $\leqslant$ defined by: $a \leqslant b$ if and only if $a^{-1} a \leqq b^{-1} b$. The Vagner-Preston representation of $S$ then embeds $S$ as a full inverse subsemigroup of $T_{S}$.

An application of these concepts is then given. Let $R$ and $S$ be inverse semigroups and let $\theta_{1}\left(\theta_{2}\right)$ be an isomorphism of a semilattice $E$ onto the semilattice of $R(S)$. Then there exists a locally uniform presemilattice $W$ and embeddings $\varphi_{1}, \varphi_{2}$ of $R$ and $S$, respectively, as full inverse subsemigroups of $T_{W}$ such that

(1) $\theta_{1} \varphi_{1}=\theta_{2} \varphi_{2}$

(2) $\left(e \theta_{1} \varphi_{1}, f \theta_{2} \varphi_{2}\right) \in \mathscr{D}$ if and only if $E e$ is isomorphic to $E f$.

\section{Basic concepts}

The reader is referred to Clifford and Preston (1961 and 1967) for the basic properties of inverse semigroups and all undefined notation and terminology.

For any set $X, \mathscr{I}_{X}$ will denote the summetric inverse semigroup on $X$. Let $E$ be a semilattice and let $T_{E}$ denote the subset of $\mathscr{I}_{E}$ consisting of those $\alpha$ for which the domain and range are principal ideals and $\alpha$ is an isomorphism of its domain onto its range. We shall denote the domain and range of a mapping $\alpha$ by $\Delta(\alpha)$ and $\nabla(\alpha)$, respectively. The semilattice $E$ is uniform if and only if any two principal ideals are isomorphic.

LEMMA 1.1 (Munn (1966)). For any semilattice $E, T_{E}$ is an inverse subsemigroup of $\mathscr{I}_{E}$ with semilattice isomorphic to $E$. Furthermore $T_{E}$ is bisimple if and only if $E$ is uniform. 
An inverse subsemigroup $S$ of an inverse semigroup $T$ is a full inverse subsemigroup if and only if $S$ contains all the idempotents of $T$. Howie (1964) showed that an inverse semigroup has a maximum idempotent separating congruence. Munn (1970) observed that this is also the maximum congruence contained in $\mathscr{H}$.

LEMMA 1.2 (Munn (1970)). Let $S$ be an inverse semigroup with semilattice $E$.

(1) For all $a \in S$, the mapping $\theta_{a}: E a a^{-1} \rightarrow E a^{-1} a$ defined by

$$
x \theta_{a}=a^{-1} x a \quad\left(x \in E a a^{-1}\right)
$$

is an isomorphism and so $\theta_{a} \in T_{E}$.

(2) The mapping $\theta: S \rightarrow T_{E}$ defined by $a \theta=\theta_{a}(a \in S)$ is a homomorphism and $\theta \circ \theta^{-1}$ is the maximum idempotent separating congruence on $S$.

(3) $S \theta$ is a full inverse subsemigroup of $T_{E}$.

We shall refer to the homomorphism $\theta$, defined in Lemma 1.2, as the Munn representation of $S$ on E. In general, of course, $\theta$ is not faithful and clearly $\theta$ is faithful if and only if the maximum idempotent separating congruence on $S$ is the identity congruence. An inverse semigroup is fundamental if and only if the maximum idempotent separating congruence on $S$ is the identity congruence. From Munn (1970), we than have the following observation.

CoROllary 1.3. Let $S$ be an inverse semigroup with semilattice $E$. Then $S$ is fundamental if and only if $S$ is isomorphic to a full inverse subsemigroup of $T_{E}$.

The Munn representation has been of great interest to those studying inverse semigroups and it is natural to ask if it is possible to inflate or enlarge the set $E$, on which $S$ is represented, in order to obtain a faithful representation of $S$ while retaining some of the features of the Munn representation. We show that, in fact, modulo a certain interpretation, one already exists and then we shall give an application of the concepts introduced.

Let $X$ be a set and $S$ an inverse subsemigroup of $\mathscr{I}_{X}$. An equivalence relation $\rho$ on $X$ will be called an $S$-congruence if and only if the following two conditions are satisfied: (1) if $x \in \Delta(a)$ then $x \rho \subseteq \Delta(a)$ and (2) if $x$ and $y \in \Delta(a)$ then $(x, y) \in \rho$ if and only if $(x a, y a) \in \rho$. A mapping $\alpha$ of $X$ onto a set $Y$ is $S$-compatible if and only if $\alpha \circ \alpha^{-1}$ is an $S$-congruence. If $\alpha: X \rightarrow Y$ is an $S$-compatible mapping then each $a \in S$ induces a mapping $a^{*}$ with domain $\{x \alpha: x \in \Delta(a)\}$ such that, for $x \alpha \in \Delta\left(a^{*}\right),(x \alpha) a^{*}=(x a) \alpha$. Moreover, the mapping $a \rightarrow a^{*}$ is clearly a homomorphism of $S$ into $\mathscr{I}_{Y}$ which we call the homomorphism induced by $\alpha$. 
Our objective is to find a set $X$ and a homomorphism $\psi$ of $S$ into $\mathscr{I}_{X}$ for which there is an $S$-compatible mapping $\xi$ of $X$ onto $E$ such that the induced homomorphism $\xi^{*}$ actually maps $S \psi$ into $T_{E}$ and composes with $\psi$ to yield the Munn representation $\theta$. See Figure 1 .
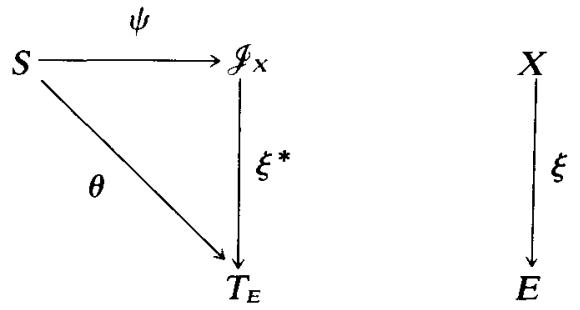

Figure 1

Viewing $X$ as an enlargement of $E$, one might if $X$ has any structure. By a pre-order on a set $A$ we shall mean any reflexive transitive relation. Let $\leqslant$ be a pre-order on a nonempty set $A$ and let $\nu=\{(a, b) \in A \times A: a \leqslant b$ and $b \leqslant a\}$. Then $\nu$ is an equivalence relation on $A$ and $A / \nu$ is a partially ordered set with respect to the induced relation

$$
a \nu \leqq b \nu \text { if and only if } a \leqslant b .
$$

We call $\leqq$ the naturally induced order on $A / \nu$. Clearly $\nu$ is the smallest equivalence relation on $A$ for which (1) defines a partial order on $A / \nu$. We call $\nu$ the minimum partial order congruence (mpo-congruence) on $A$ and say that $A$ is a presemilattice if and only if $A / \nu$ is a semilattice with respect to the naturally induced order. Clearly, then, $X$ above would be a presemilattice with respect to the relation $\leqslant$ defined by

$$
x \leqslant y \text { if and only if } x \xi=y \xi .
$$

So we begin with some comments on presemilattices.

Let $X$ be a presemilattice with pre-order $\leqslant$ and mpo-congruence $\nu$. Clearly $X$ is a semilattice with respect to $\leqslant$ if and only if $\nu$ is the identity relation. A subset $A$ of $X$ is an ideal of $X$ provided that $x \leqslant y$ and $y \in A$ implies that $x \in A$. In particular, if $A$ is an ideal and $x \in A$ then $x \nu \subseteq A$. Also $A$ is clearly a presemilattice with respect to the restriction of the pre-order on $X$ to $A$ and the mpo-congruence on $A$ is just the restriction to $A$ of the mpocongruence on $X$. For $a \in X$, we call $\{x \in X: x \leqslant a\}$ the principal ideal generated by $a$ and denote it by $\langle a\rangle$.

A bijection $\alpha$ of one pre-ordered set $A$ onto another $B$ will be called an isomorphism provided that, for all $a, b \in A, a \leqslant b$ if and only if $a \alpha \leqslant b \alpha$. In 
particular, if $\nu_{A}$ and $\nu_{B}$ denote the respective mpo-congruences then $(a, b) \in$ $\nu_{A}$ if and only if $(a \alpha, b \alpha) \in \nu_{B}$.

Let $T_{X}$ denote the subset of $\mathscr{I}_{X}$ consisting of those mappings $\alpha$ for which the domain and the range are both principal ideals and $\alpha$ is an isomorphism. Note that the use of the notation $T_{X}$ does not conflict with the notation introduced by Munn and described above since, in the event that $X$ is a semilattice, $T_{X}$ as defined here is the same object as introduced by Munn. The verification of the following lemma is entirely routine.

LEMMA 1.4. (1) $T_{X}$ is an inverse subsemigroup of $\Phi_{X}$ with semilattice isomorphic to $X / \nu$.

(2) $\nu$ is a $T_{X}$-congruence.

For $x \in X$ we denote by $\varepsilon_{x}$ the identity mapping on $\langle x\rangle$. Then $\varepsilon_{x}^{2}=$ $\varepsilon_{x} \in T_{X}$. On the other hand every idempotent of $T_{X}$ is clearly of this form, although the representation may not be unique since $\varepsilon_{x}=\varepsilon_{y}$ if and only if $(x, y) \in \nu$.

Extending the terminology introduced by Munn (1970) we shall say that $X$ is uniform if and only if $\langle x\rangle$ is isomorphic to $\langle y\rangle$, for all $x, y \in X$ and that $X$ is locally uniform if $|x \nu|=|y \nu|$, for all $x, y \in X$. Let $E=X / \nu$. Let $x, y \in X$ $(x, y) \in \nu, \alpha \in T_{X}$ and $x \nu \subseteq \Delta(\alpha)$. Then $(x \alpha, y \alpha) \in \nu$. Hence, if $\Delta(\alpha)=\langle a\rangle, \alpha$ induces a mapping $\alpha^{\prime}$ of $E$ which can be defined as follows:

(1) $\Delta\left(\alpha^{\prime}\right)=\{x \nu: x \in \Delta(\alpha)\}=\langle a \nu\rangle$;

(2) $(x \nu) \alpha^{\prime}=(x \alpha) \nu \quad\left(x \nu \in \Delta\left(\alpha^{\prime}\right)\right)$.

Since $\alpha$ is an isomorphism, $\alpha^{\prime}$ is well defined. Also the domain of $\alpha^{\prime}$ is a principal ideal, by definition, while the range of $\alpha^{\prime}$ is clearly $\langle(a \alpha) \nu\rangle$, a principal ideal. Let $x \nu, y \nu \in \Delta\left(\alpha^{\prime}\right)$. Then $x, y \in \Delta(\alpha)$ and $x \nu \leqq y \nu$ if and only if $x \leqslant y$. Since $\alpha$ is an isomorphism, $x \leqslant y$ if and only if $x \alpha \leqslant y \alpha$, that is, if and only if $(x \alpha) \nu \leqq(y \alpha) \nu$. Thus $\alpha^{\prime}$ is an isomorphism and so $\alpha^{\prime} \in T_{E}$.

LEMMA 1.5. The mapping $\pi: \alpha \rightarrow \alpha^{\prime}$ of $T_{X}$ into $T_{E}$ is a homomorphism of $T_{X}$ onto a full inverse subsemigroup of $T_{E}$ such that $\pi^{\circ} \pi^{-1}$ is the maximum idempotent separating congruence on $T_{X}$. Furthermore $\pi$ is an epimorhism if and only if $|x \nu|=|y \nu|$ whenever $\langle x \nu\rangle$ and $\langle y \nu\rangle$ are isomorphic ideals $(x, y \in$ $X)$. In particular, $\pi$ is an epimorphism if $X$ is locally uniform.

Proof. Clearly $\pi$ is a homomorphism. Let $x \nu \in E$. Then $\varepsilon_{x}^{\prime}$ is the identity mapping on $\langle x \nu\rangle$. Hence $\pi$ maps the idempotents of $T_{X}$ onto those of $T_{E}$ and $T_{X} \pi$ is a full inverse subsemigroup of $T_{E}$. On the other hand, if $\varepsilon_{x} \neq \varepsilon_{y}$ then $(x, y) \notin \nu$ while $\Delta\left(\varepsilon_{x}^{\prime}\right)=\langle x \nu\rangle$ and $\Delta\left(\varepsilon_{y}^{\prime}\right)=\langle y \nu\rangle$. Therefore $\varepsilon_{x}^{\prime} \neq \varepsilon_{y}^{\prime}$ and $\pi$ 
is idempotent separating. However, by Corollary $1.3, T_{X} \pi$ is fundamental and so $\pi \circ \pi^{-1}$ must be the maximum idempotent separating congruence on $\cdot T_{X}$.

Now suppose that $|x \nu|=|y \nu|$ whenever $\langle x \nu\rangle$ and $\langle y \nu\rangle$ are isomorphic. Let $\beta \in T_{E}$. Let $\Delta(\beta)=\langle y \nu\rangle$, for some $y \in X$. For each $x \nu \in \Delta(\beta),\langle x \nu\rangle$ is isomorphic to $\langle x \nu \beta\rangle$ and so let $\beta_{x \nu}$ be a bijection of $x \nu$ onto $x \nu \beta$. Let $\alpha$ be the mapping defined as follows:

(1) $\Delta(\alpha)=\langle y\rangle$;

(2) $x \alpha=x \beta_{x \nu} \quad(x \in \Delta(\alpha))$.

It is straightforward to verify that $\alpha \in T_{X}$ and that $\alpha^{\prime}=\beta$. Thus $\pi$ is an epimorphism.

Conversely, if $\pi$ is an epimorphism and $\langle x \nu\rangle$ is isomorphic to $\langle y \nu\rangle$ then there exists an isomorphism $\beta$, say, of $x \nu$ onto $y \nu$. Let $\alpha \in T_{x}$ be such that $\alpha^{\prime}=\beta$. Then $x \alpha \in y \nu$ and so the restriction of $\alpha$ to $x \nu$ is a bijection of $x \nu$ onto $y \nu$. Thus $|x v|=|y v|$.

We shall refer to $\pi$ as the natural projection of $T_{X}$ into $T_{E}$ and, for each $\alpha \in T_{X}$, we shall refer to $\alpha^{\prime}=\alpha \pi$ as the projection of $\alpha$ in $T_{E}$.

LemMa 1.6. The following statements are equivalent.

(1) $T_{X}$ is bisimple.

(2) $X$ is uniform.

(3) $X$ is locally uniform and $E=X / \nu$ is uniform.

Proof. Suppose that $T_{X}$ is bisimple. Let $x, y \in X$. Then there exists an element $\alpha \in T_{X}$ with $\alpha \alpha^{-1}=\varepsilon_{x}$ and $\alpha^{-1} \alpha=\varepsilon_{y}$; that is, $\Delta(\alpha)=\langle x\rangle$ and $\nabla(\alpha)=$ $\langle y\rangle$. Hence $\langle x\rangle$ is isomorphic to $\langle y\rangle$ and $X$ is uniform. Thus (1) implies (2).

Now let $X$ be uniform and $x, y \in X$. Then there is an isomorphism $\alpha \in$ $T_{x}$ with domain equal to $\langle x\rangle$ and range equal to $\langle y\rangle$. Therefore $(x v) \alpha=y v$ and $|x \nu|=|y \nu|$. Thus $X$ is locally uniform. Furthermore, the projection $\alpha^{\prime}$ of $\alpha$ in $T_{E}$ has domain $\langle x \nu\rangle$ and range $\langle y \nu\rangle$. Hence $E$ is uniform. Thus (2) implies (3).

Finally, let (3) hold. By Lemma 1.1, $T_{E}$ is bisimple. Since $X$ is locally uniform, it follows from Lemma 1.5, that the natural projection $\pi$ of $T_{X}$ is an idempotent separating homomorphism of $T_{X}$ onto $T_{E}$. Hence $T_{X}$ is bisimple.

Let $E$ be a semilattice and $U$ be a nonempty set. Then the relation $\leqslant$ defined on $U \times E$ by

$$
(u, e) \leqslant(v, f) \text { if and only if } e \leqq f
$$

is a pre-order on $U \times E$ which we call the natural pre-order. The mpocongruence $\nu$ on $U \times E$ is such that 


$$
((u, e),(v, f)) \in \nu \text { if and only if } e=f \text {. }
$$

Thus $U \times E / \nu$ is clearly isomorphic to $E$ and $U \times E$ is a presemilattice. Also $U \times E$ is locally uniform. Hence $U \times E$ is uniform (and $T_{U \times E}$ is bisimple) if and only if $E$ is uniform.

Lemma 1.7. Let $X$ be locally uniform and $x_{0} \in X$. Let $U=x_{0} \nu$ and $E=$ $X / \nu$. Then $X$ is isomorphic to $U \times E$ under the natural pre-order and $T_{X}$ is isomorphic to $T_{U \times E}$.

Proof. For each $x \nu \in E$ let $f_{x \nu}$ be a bijection of $x \nu$ onto $x_{0} \nu$. Since $X$ is locally uniform such bijections exist. Define $\varphi: X \rightarrow U \times E$ by

$$
x \varphi=\left(x f_{x \omega}, x \nu\right)
$$

It is clear that $\varphi$ is an isomorphism and the result follows.

Since a locally uniform presemilattice decomposes nicely, by Lemma 1.7, into the product of a set and a semilattice we now relate an arbitrary presemilattice to a locally uniform semilattice. If $X$ is a presemilattice with mpocongruence $\nu$ we call $X / \nu$ the semilattice of $X$.

Lemma 1.8. Let $X$ be a presemilattice. Then $X$ can be embedded in a locally uniform presemilattice $Y$ in such a way that the semilattice of $Y$ is isomorphic to the semilattice of $X$. Moreover, the embedding can be chosen so that $T_{X}$ can be embedded as a full inverse subsemigroup of $T_{Y}$, in a natural way.

Proof. Let $Z$ be any set such that $|Z|=|X|$, if $X$ is infinite, and countably infinite if $X$ is finite. Let $Y=Z \times X$ and define the relation $\leqslant$ on $Y$ by

$$
\left(z_{1}, x_{1}\right) \leqslant\left(z_{2}, x_{2}\right) \text { if and only if } x_{1} \leqslant x_{2} .
$$

Then $\leqslant$ is a pre-order on $Y$. Let $\nu_{X}\left(\nu_{Y}\right)$ be the mpo-congruence on $X(Y)$. Then, for any $x \in X, z \in Z$, it is easily seen that $(z, x) \nu_{Y}=Z \times x \nu_{X}$. Hence the mapping $(z, x) \nu_{Y} \rightarrow x \nu_{X}$ is an isomorphism of the semilattice $Y / \nu_{Y}$ of $Y$ onto the semilattice $X / \nu_{X}$ of $X$. Also since, for all $z \in Z, x \in X,\left|(z, x) \nu_{Y}\right|=$ $\left|Z \times x \nu_{X}\right|=|Z|, Y$ is locally uniform.

Let $z_{0} \in Z$. Then the set of elements of the form $\left(z_{0}, x\right)$ where $x \in X$ is clearly a presemilattice contained in $Y$ isomorphic to $X$. For convenience identify this presemilattice with $X$. We now show that we can embed $T_{X}$ in $T_{Y}$ in such a way that the restriction of the action of the embedded elements to $X$ (really $\left\{z_{0}\right\} \times X$ ) is just the action of the original elements of $T_{X}$.

For each $\alpha \in T_{X}$ let $\alpha^{+}$be the mapping defined as follows:

(1) $\Delta\left(\alpha^{+}\right)=\{(z, x): x \in \Delta(\alpha)\}$

(2) $(z, x) \alpha^{+}=(z, x \alpha) \quad\left((z, x) \in \Delta\left(\alpha^{+}\right)\right)$. 
Then $\alpha^{+} \in T_{Y}$ and the mapping $\alpha \rightarrow \alpha^{+}$is an embedding of $T_{X}$ as a full inverse subsemigroup of $T_{Y}$ with the desired properties.

\section{Decomposition of $T_{x}$}

If $X$ is a locally uniform presemilattice then we saw, in Lemma 1.8 , that $X$ is isomorphic to the product of any one of its mpo-congruence classes with the semilattice of $X$ under the natural pre-order. In this section we show that in these circumstances $T_{X}$ also decomposes. For this purpose we introduce a variant of the wreath product construction.

Let $U$ and $F$ be sets. Let $A$ be an inverse subsemigroup of $\mathscr{I}_{F}$ and $H$ be a subgroup of the symmetric group, $S_{U}$, on $U$. Let $W$ denote the set of elements $\alpha$ in $\mathscr{I}_{U \times F}$ for which there exists an $\alpha^{\prime} \in A$ and, for each $e \in \Delta\left(\alpha^{\prime}\right)$, an $\alpha_{e} \in H$ with

(1) $\Delta(\alpha)=\left\{(u, e): e \in \Delta\left(\alpha^{\prime}\right)\right\}$;

(2) $(u, e) \alpha=\left(u \alpha_{e}, e \alpha^{\prime}\right) \quad((u, e) \in \Delta(\alpha))$.

Let $\alpha^{*}$ denote the mapping $e \rightarrow \alpha_{e}$ of $\Delta\left(\alpha^{\prime}\right)$ into $H$. Then $\alpha$ is completely determined by the pair $\left(\alpha^{*}, \alpha^{\prime}\right)$ and we identify $\alpha$ with this pair.

On the other hand, given an $\alpha^{\prime} \in \mathscr{I}_{F}$ and a mapping $\alpha^{*}: e \rightarrow \alpha_{e}$ of $\Delta\left(\alpha^{\prime}\right)$ into $H$ we can use (1) and (2) above to define an element $\alpha$ in $W$. Thus we can consider $W$ as consisting of all such pairs. As elements of $\mathscr{I}_{U \times F}$ we can multiply elements of $W$ and we shall see that such products fall into $W$.

Let $\alpha=\left(\alpha^{*}, \alpha^{\prime}\right)$ and $\beta=\left(\beta^{*}, \beta^{\prime}\right)$ be elements of $W$. Then

$$
\begin{aligned}
\Delta(\alpha \beta) & =\{(u, e):(u, e) \in \Delta(\alpha),(u, e) \alpha \in \Delta(\beta)\} \\
& =\left\{(u, e): e \in \Delta\left(\alpha^{\prime}\right), e \alpha^{\prime} \in \Delta\left(\beta^{\prime}\right)\right\} \\
& =\left\{(u, e): e \in \Delta\left(\alpha^{\prime} \beta^{\prime}\right)\right\} .
\end{aligned}
$$

Furthermore, for $(u, e) \in \Delta(\alpha \beta)$,

$$
\begin{aligned}
(u, e) \alpha \beta & =\left(u \alpha_{e}, e \alpha^{\prime}\right) \beta \\
& =\left(u \alpha_{e} \beta_{e \alpha^{\prime}}, e \alpha^{\prime} \beta^{\prime}\right) .
\end{aligned}
$$

Thus $\alpha \beta=\left(\gamma^{*}, \gamma^{\prime}\right)$ where

$$
\gamma^{\prime}=\alpha^{\prime} \beta^{\prime} \text { and } e \gamma^{*}=\left(e \alpha^{*}\right)\left(e \alpha^{\prime}\right) \beta^{*} \quad\left(e \in \Delta\left(\alpha^{\prime} \beta^{\prime}\right)\right) .
$$

Therefore $\alpha \beta \in W$ and $W$ is a subsemigroup of $\mathscr{I}_{U \times F}$.

LeMma 2.1. $W$ is an inverse subsemigroup of $\mathscr{I}_{U \times F}$. The element $\alpha=$ $\left(\alpha^{*}, \alpha^{\prime}\right)$ is an idempotent if and only if $\alpha^{\prime}$ is an idempotent and $e \alpha^{*}$ is the 
identity of $H$ for all $e \in \Delta\left(\alpha^{\prime}\right)$. Thus the semilattice of $W$ is isomorphic to the semilattice of $A$. If $\beta=\left(\beta^{*}, \beta^{\prime}\right) \in W$ and $\mathscr{G}$ denotes any one of Green's relations then $(\alpha, \beta) \in \mathscr{G}$ if and only if $\left(\alpha^{\prime}, \beta^{\prime}\right) \in \mathscr{G}$.

Proof. We have already seen that $W$ is a subsemigroup of $\mathscr{I}_{U \times F}$. Let $\alpha=\left(\alpha^{*}, \alpha^{\prime}\right) \in W$. Then one can easily verify that $\alpha^{-1}$, as an element of $\mathscr{I}_{U_{\times F}}$, is just $\left(\beta^{*}, \beta^{\prime}\right)$ where $\beta^{\prime}=\left(\alpha^{\prime}\right)^{-1}$ and, for all $f \in \Delta\left(\beta^{\prime}\right)=\Delta\left(\left(\alpha^{\prime}\right)^{-1}\right)=\nabla\left(\alpha^{\prime}\right)$, $f \beta^{*}=\left(e \alpha^{*}\right)^{-1}$, where $e$ is the unique element of $\Delta\left(\alpha^{\prime}\right)$ such that $f=e \alpha^{\prime}$. Thus $W$ is an inverse subsemigorup of $\mathscr{I}_{U \times F}$.

The conditions for $\alpha=\left(\alpha^{*}, \alpha^{\prime}\right)$ to be an idempotent follow easily from (2).

Let $\alpha=\left(\alpha^{*}, \alpha^{\prime}\right), \beta=\left(\beta^{*}, \beta^{\prime}\right)$ be any elements of $W$. Let $\alpha^{-1}=$ $\left(\gamma^{*},\left(\alpha^{\prime}\right)^{-1}\right), \beta^{-1}=\left(\delta^{*},\left(\beta^{\prime}\right)^{-1}\right)$, say. Then

$$
\alpha \alpha^{-1}=\left(\kappa, \alpha^{\prime}\left(\alpha^{\prime}\right)^{-1}\right) \text { and } \beta \beta^{-1}=\left(\lambda, \beta^{\prime}\left(\beta^{\prime}\right)^{-1}\right)
$$

where, since $\alpha \alpha^{-1}\left(\beta \beta^{-1}\right)$ is an idempotent, $\kappa(\lambda)$ maps $\Delta\left(\alpha^{\prime}\left(\alpha^{\prime}\right)^{-1}\right)$ $\left(\Delta\left(\beta^{\prime}\left(\beta^{\prime}\right)^{-1}\right)\right)$ onto the identity of $H$. Hence $\alpha \alpha^{-1}=\beta \beta^{-1}$ if and only if $\alpha^{\prime}\left(\alpha^{\prime}\right)^{-1}=\beta^{\prime}\left(\beta^{\prime}\right)^{-1}$. In other words $\alpha \mathscr{R} \beta$ if and only if $\alpha^{\prime} \mathscr{R} \beta^{\prime}$. Similar arguments can be applied to the relations $\mathscr{L}, \mathscr{H}, \mathscr{D}$ and $\mathscr{I}$.

Corollary 22. W is bisimple if and only if $A$ is bisimple.

Since the construction of $W$ is analogous to that of wreath products we write $W=(H, U)$ wr $(A, F)$.

We are particularly interested in the case $H=S_{U}, A=T_{E}$, where $U$ is a set and $E$ is a semilattice. Let $U \times E$ be endowed with the natural pre-order. Then we have the following result decomposing $T_{U \times E}$.

THEOREM 2.3. $T_{U \times E}=\left(S_{U}, U\right) w r\left(T_{E}, E\right)$.

Proof. Let $W=\left(S_{U}, U\right) \operatorname{wr}\left(T_{E}, E\right)$ and let $\alpha=\left(\alpha^{*}, \alpha^{\prime}\right) \in W$. If $\Delta\left(\alpha^{\prime}\right)=$ $\langle e\rangle$ and $u_{0} \in U$ then

$$
\begin{aligned}
\Delta(\alpha) & =\left\{(u, f): f \in \Delta\left(\alpha^{\prime}\right)\right\}=\{(u, f): f \leqq e\} \\
& =\left\{(u, f):(u, f) \leqslant\left(u_{0}, e\right)\right\}=\left\langle\left(u_{0}, e\right)\right\rangle
\end{aligned}
$$

Thus $\Delta(\alpha)$ is a principal ideal and also $\nabla(\alpha)=\Delta\left(\alpha^{-1}\right)$. Let $(a, f),(b, g) \in \Delta(\alpha)$. Then $(a, f) \alpha=\left(a\left(f \alpha^{*}\right), f \alpha^{\prime}\right)$ and $(b, g) \alpha=\left(b\left(g \alpha^{*}\right), g \alpha^{\prime}\right)$. Now $(a, f) \leqslant(b, g)$ if and only if $f \leqq g$, while $(a, f) \alpha \leqslant(b, g) \alpha$ if and only if $f \alpha^{\prime} \leqq g \alpha^{\prime}$. But, since $\alpha^{\prime} \in T_{E} \cdot f \leqq g$ if and only if $f \alpha^{\prime} \leqq g \alpha^{\prime}$. Therefore $(a, f) \leqslant(b, g)$ if and only if $(a, f) \alpha \leqslant(b, g) \alpha$. Thus $\alpha$ is an order isomorphism of $\Delta(\alpha)$ onto $\nabla(\alpha)$ and $\alpha \in T_{U \times E}$. Hence $W \subseteq T_{U \times E}$.

We now consider the converse inclusion. Let $\nu$ denote the mpo- 
congruence on $U \times E$. Thus $\nu=\{((u, f),(v, g)): f=g\}$ and $(u, f) \nu \rightarrow f$ is an isomorphism of $U \times E / \nu$ onto $E$. If we identify $U \times E / \nu$ with $E$ via this isomorphism then the natural projection $\pi: T_{U \times E} \rightarrow T_{E}$ of Lemma 1.5 can be described as follows: for $\alpha \in T_{U \times E}$

(1) $\Delta(\alpha \pi)=\{e: U \times\{e\} \subseteq \Delta(\alpha)\}$,

(2) $e(\alpha \pi)=f$, where $(U \times\{e\}) \alpha=U \times\{f\},(e \in \Delta(\alpha \pi))$.

For $\alpha \in T_{U \times E}$ and $e \in \Delta(\alpha \pi)$ define a mapping $\alpha_{e}: U \rightarrow U$ by $(u, e) \alpha=$ $\left(u \alpha_{e}, e(\alpha \pi)\right)$, for all $u \in U$. Since the restriction of $\alpha$ to $U \times\{e\}$ is a bijection of $U \times\{e\}$ onto $U \times\{e \alpha \pi\}$, it follows that $\alpha_{e} \in S_{U}$. Now define $\alpha^{*}: \Delta(\alpha \pi) \rightarrow S_{U}$ by $e \alpha^{*}=\alpha_{e}$. Then clearly $\beta=\left(\alpha^{*}, \alpha \pi\right) \in W$. But,

$$
\begin{aligned}
\Delta(\beta) & =\{(u, e): e \in \Delta(\alpha \pi)\}=\{(u, e): U \times\{e\} \subseteq \Delta(\alpha)\} \\
& =\Delta(\alpha)
\end{aligned}
$$

and, for $(u, e) \in \Delta(\alpha)=\Delta(\beta)$,

$$
\begin{aligned}
(u, e) \beta & =\left(u\left(e \alpha^{*}\right), e(\alpha \pi)\right) \\
& =\left(u \alpha_{e}, e(\alpha \pi)\right) \\
& =(u, e) \alpha
\end{aligned}
$$

Thus $\alpha=\beta \in W$ and $T_{U \times E} \subseteq W$, as required.

\section{An inflated Munn representation}

In this section we consider the first application of the concepts developed above. We show that there does exist an inflated Munn representation and that, in fact, with the appropriate interpretation the Vagner-Preston representation will serve for this purpose.

Let $S$ be an inverse semigroup. Defines the relation $\leqslant$ on $S$ by: $a \leqslant b$ if and only if $a^{-1} a \leqq b^{-1} b$. Then $\leqslant$ is clearly a pre-order on $S$ for which the mpo-congruence is $\nu=\mathscr{L}$. Hence $S / \nu=S / \mathscr{L}$, under the naturally induced order from $\leqslant$, is just the set of $\mathscr{L}$-classes of $S$ under the usual partial ordering of the $\mathscr{L}$-classes of an inverse semigroup and so is isomorphic to the semilattice of $S$. Hence $S$ is a presemilattice under $\leqslant$.

Let $\rho: a \rightarrow \rho_{a}$ mapping $S$ into $\mathscr{I}_{S}$ be the Vagner-Preston representation of $S$. Then

(1) $\Delta\left(\rho_{a}\right)=S a a^{-1}$

(2) $x \rho_{a}=x a \quad\left(x \in S a a^{-1}\right)$. 
Considering $S$ as a presemilattice as above, $\Delta\left(\rho_{a}\right)=S a a^{-1}=\left\langle a^{-1}\right\rangle$, a principal ideal of $S$, as is $\nabla\left(\rho_{a}\right)=S a^{-1} a=\langle a\rangle$. Also, for $x, y \in \Delta\left(\rho_{a}\right), x^{-1} x, y^{-1} y \leqq a a^{-1}$. Now $x \leqslant y$ if and only if $x^{-1} x \leqq y^{-1} y$ while $x a \leqslant y a$ if and only if $a^{-1} x^{-1} x a=$ $(x a)^{-1}(x a) \leqq(y a)^{-1}(y a)=a^{-1} y{ }^{-1} y a$. But, since $x^{-1} x, y^{-1} y \leqq a a^{-1}$ it follows that $x^{-1} x \leqq y^{-1} y$ if and only if $a^{-1} x^{-1} x a \leqq a^{-1} y^{-1} y a$. Therefore $x \leqslant y$ if and only if $x a \leqslant y a$. Thus $\rho_{a}$ is an isomorphism of $\left\langle a^{-1}\right\rangle$ onto $\langle a\rangle$ and $S \rho \subseteq T_{s}$. We are now part of the way towards proving the following theorem.

THEOREM 3.1. Let $S$ be an inverse semigroup and define the relation $\leqslant$ on $S$ by $a \leqslant b$ if and only if $a^{-1} a \leqq b^{-1} b$. Then $\leqslant$ is a pre-order on $S$ with respect to which $S$ is a presemilattice. The Vagner-Preston representation $\rho$ of $S$ embeds $S$ as a full inverse subsemigroup of $T_{S}$.

If $\nu$ is the mpo-congruence on $S$ then $\nu=\mathscr{L}$ and $S / \nu$ is isomorphic to the semilattice $E$ of $S$. Moreover, the mapping $\xi: a \rightarrow a^{-1} a$ is an $S$-compatible mapping of $S$ onto $E$ such that $\rho \xi^{*}$ is the Munn representation of $S$ on $E$.

Proof. It remains to show that $S \rho$ is a full inverse subsemigroup of $T_{S}$ and to verify the assertions regarding $\xi$.

Let $\varepsilon$ be an idempotent of $T_{s}$ with domain $\langle a\rangle, a \in S$. Let $e=a^{-1} a$. Then $\varepsilon$ and $\rho_{e}$ are both identity mappings on $\langle a\rangle$. Thus $\varepsilon=\rho_{e}$ and $S \rho$ is a full inverse subsemigroup of $T_{s}$.

For $a, b \in S$ we have that $(a, b) \in \xi \circ \xi^{-1}$ if and only if $a^{-1} a=b^{-1} b$; that is, if and only if $(a, b) \in \nu$. Hence $\xi \circ \xi^{-1}=v$, a $T_{s}$-congruence and therefore an $S$-congruence. Hence $\xi$ is an $S$-compatible mapping and clearly maps $S$ onto $E$.

Let $a \in S$ and consider $\alpha=a \rho \xi^{*}$. Since $S \xi=E$, we know that $\alpha \in \mathscr{I}_{E}$. By the definition of $\xi^{*}$,

$$
\begin{aligned}
\Delta(\alpha) & =\{x \xi: x \in \Delta(a \rho)\}=\left\{x \xi: x^{-1} x \leqq a a^{-1}\right\} \\
& =\left\{x^{-1} x: x^{-1} x \leqq a a^{-1}\right\}=\Delta\left(\theta_{a}\right)
\end{aligned}
$$

where we continue to denote by $\theta: a \rightarrow \theta_{a}$ the Munn representation of $S$ on E. Also, for $x \xi \in \Delta(\alpha)(x \in S)$,

$$
(x \xi) \alpha=(x \xi)\left(a \rho \xi^{*}\right)=(x(a \rho)) \xi=(x a) \xi=a^{-1} x^{-1} x a .
$$

Thus

$$
\left(x^{-1} x\right) \alpha=a^{-1} x^{-1} x a=\left(x^{-1} x\right) \theta_{a} .
$$

Hence $\alpha=\theta_{a}$ and $\rho \xi^{*}=\theta$, as required.

Corollary 3.2. Let $S$ be an inverse semigroup with semilattice E. Then there exists a set $U$ and an embedding $\varphi$ of $S$ as a full inverse subsemigroup of 
$T_{U \times E^{\prime}}$, where $U \times E$ is endowed with the natural pre-order, such that the mapping $\eta:(u, e) \rightarrow e$ is $S \varphi$-compatible and $\varphi \eta^{*}$ is the Munn representation.

Proof. By Theorem 3.1, $S$ can be embedded as a full inverse subsemigroup of $T_{\mathrm{S}}$, where $S$ has an appropriate pre-order and the semilattice of $S$, as a presemilattice, is isomorphic to $E$. Denote this embedding by $\rho$ and let $\xi, \xi *$ be as in Theorem 3.1. But for the assertion regarding $\eta$ the result would now follow from the fact that the semilattice of $S$, as a pre-ordered set, is $E$ by invoking Lemma 1.7 and Lemma 1.8. However, to establish the assertion regarding $\eta$ we must follow this process in more detail.

As in Lemma 1.8 let $Y=Z \times S$ under the pre-order induced from $S$. If $z_{0} \in Z$, let $\sigma$ be the embedding $s \rightarrow\left(z_{0}, s\right)$ of $S$ in $Y$ and let $\sigma^{*}$ be the embedding of $T_{S}$ in $T_{Y}$ where, for $\alpha \in T_{s}$, the domain of $\alpha \sigma^{*}$ is $\{(a, s): s \in$ $\Delta(\alpha)\}$ and, for $(z, s)$ in the domain of $\alpha \sigma^{*},(z, s) \alpha \sigma^{*}=(z, s \alpha)$. Let $\xi_{1}$ be the mapping $(z, a) \rightarrow a^{-1} a$, of $Y$ onto $E$. Then $\xi_{1} \circ \xi_{1}^{-1}$ is the mpo-congruence on $Y$ and so is a $T_{\gamma}$-congruence. It is evident that $\sigma \xi_{1}=\xi$ and also that $\sigma^{*} \xi_{1}^{*}=\xi^{*}$.
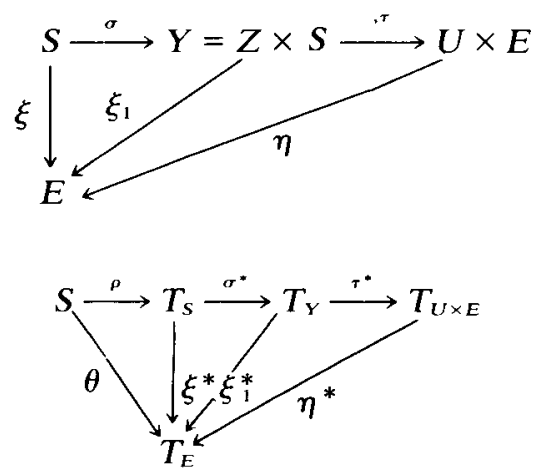

Let $\nu$ be the mpo-congruence on $Y, y_{0} \in Y$ and $U^{*}=y_{0} \nu$. for each $y \nu \in Y / \nu$ let $f_{y \nu}$ be a bijection of $y \nu$ onto $y_{0} \nu$. Define $\tau: Y \rightarrow U \times E$ as follows: for $y=(z, a) \in Y$

$$
(z, a) \tau=\left(z f_{y \nu}, a^{1} a\right) .
$$

This is entirely similar to Lemma 1.7 and it is straightforward to show that $\tau$ is an isomorphism of $Y$ onto $U \times E$ under the naturally induced pre-order inducing an isomorphism $\tau^{*}: T_{Y} \rightarrow T_{U \times E}$. Let $\eta: U \times E \rightarrow E$ be the mapping such that $(u, e) \rightarrow e$. Then $\eta^{\circ} \eta^{-1}$ is the mpo-congruence on $U \times E$ and so $\eta$ is $T_{U \times E}$-compatible. Also $\tau \eta=\xi_{1}$ and $\tau^{*} \eta^{*}=\xi_{1}^{*}$. Let $\varphi=\rho \sigma^{*} \tau^{*}$. Since each of the embeddings $\rho, \sigma^{*}$ and $\tau^{*}$ embeds its domain as a full inverse subsemigroup of its image, $\varphi$ embeds $S$ as a full inverse subsemigroup of $T_{U \times E}$. Finally, $\varphi \eta^{*}=\rho \sigma^{*} \tau^{*} \eta^{*}=\rho \sigma^{*} \xi_{1}^{*}=\rho \xi^{*}=\theta$, the Munn representation. 


\section{An application}

In the two results of this section we answer the following questions that were raised with the author by T. E. Hall while the author was a visitor at Monash University in 1971 and answered by the author at that time using wreath product techniques. Since then Hall (1975) and Howie (1975) have shown how to embed inverse semigroup amalgams and semigroup amalgams over inverse semigroup cores. T. E. Hall (letter to the author) has pointed out how the amalgamation theory can also be applied to yield the results in this section.

Proposition 4.1. Let $S$ be an inverse semigroup with semilattice $E$. Then there exists an inverse semigroup $T$ and a monomorphism $\varphi$ of $S$ onto a full inverse subsemigroup of $T$ such that

(1) for $e, f \in E,(e \varphi, f \varphi) \in \mathscr{D}$ if and only if Ee is isomorphic to Ef;

(2) $T$ is bisimple if and only if $E$ is uniform.

Proof. Let $U$ and the embedding $\varphi$ of $S$ into $T_{U \times E}$ be as in Corollary 3.2. Let $e, f \in E, u \in U, x=(u, e)$ and $y=(u, f)$. Then $e \varphi$ is the identity mapping on $\langle x\rangle$, that is, $e \varphi=\varepsilon_{x}$. Similarly $f \varphi=\varepsilon_{y}$. Now $U \times E$ is locally uniform. Hence, by Lemma $1.6,\left(\varepsilon_{x}, \varepsilon_{y}\right) \in \mathscr{D}$ if and only if there is an isomorphism $\langle x \nu\rangle \rightarrow\langle y \nu\rangle$ in $T_{F}$, where $\nu$ is the mpo-congruence on $U \times E$ and $F=(U \times E) / \nu$. But $(u, e) \nu \rightarrow e$ is an isomorphism of $F=(U \times E) / \nu$ onto $E$. Hence $\langle x \nu\rangle$ is isomorphic to $\langle y \nu\rangle$ if and only if $E e$ is isomorphic to $E f$. Thus $(e \varphi, f \varphi)=\left(\varepsilon_{x}, \varepsilon_{y}\right) \in \mathscr{D}$ if and only if $E e$ is isomorphic to $E f$.

The second statement is an immediate consequence of the first.

The next result is really a special case of the more general amalgamation results of Hall (1975) and Howie (1975). However since the object constructed to obtain the result is much simpler than those introduced by Hall and Howie, we feel that it is worthy of separate consideration.

Proposition 4.2. Let $E$ be a semilattice, $R$ and $S$ inverse semigroups. Let $\theta_{1}: E \rightarrow R$ and $\theta_{2}: E \rightarrow S$ be monomorphisms of $E$ onto the semilattices of $R$ and $S$, respectively. Then there exists an inverse semigroup $T$ and monomorphisms $\psi_{1}: R \rightarrow T, \psi_{2}: S \rightarrow T$ of $R$ and $S$ onto full inverse subsemigroups of $T$ such that $\theta_{1} \psi_{1}=\theta_{2} \psi_{2}$.

Proof. Let $F$ denote the semilattice of $R$. By Corollary 3.2 there is a set $U$ and a monomorphism $\varphi_{1}: R \rightarrow T_{U \times F}$ where $U \times F$ is given the natural pre-order. Now $(u, f) \rightarrow(u, e)$ where $e \theta_{1}=f$ is an isomorphism of $U \times F$ onto $U \times E$ with the natural pre-order and induces an isomorphism $\chi_{1}: T_{U \times F} \rightarrow T_{U \times E}$. Further $\varphi_{1}^{\prime}=\varphi_{1} \chi_{1}$ is such that $e \theta_{1} \varphi_{1}^{\prime}=\varepsilon_{(u, e)}$, for any $u \in U$. 
Similarly there is a set $V$ and a monomorphism $\varphi_{2}^{\prime}$ of $S$ onto a full inverse subsemigroup of $T_{V \times E}$ where $V \times E$ is endowed with the natural pre-order and where $e \theta_{2} \varphi_{2}^{\prime}=\varepsilon_{(v, e)}$, for any $v \in V$.

Now let $\leqslant$ be the natural pre-order on $(U \times V) \times E$ and let $\xi_{1}: T_{U \times E} \rightarrow T_{(U \times V) \times E}$ be defined by

(1) $\Delta\left(\alpha \xi_{1}\right)=\{((u, v), e):(u, e) \in \Delta(\alpha)\}$,

(2) for $((u, v), e) \in \Delta\left(\alpha \xi_{1}\right),((u, v), e)\left(\alpha \xi_{1}\right)=\left(\left(u^{\prime}, v\right), e^{\prime}\right)$ where $(u, e) \alpha=$ $\left(u^{\prime}, e^{\prime}\right)$.

Then clearly $\xi_{1}$ embeds $T_{t \times E}$ as a full inverse subsemigroup of $T_{(U \times V) \times E}$. Let $\xi_{2}: T_{V \times E} \rightarrow T_{\left(U \times v^{\prime} \times E\right.}$ be defined in an analogous manner. Let $\psi_{i}=\varphi_{i}^{\prime} \xi_{i}$ $(i=1,2)$. Then $\psi_{1}\left(\psi_{2}\right)$ embeds $R(S)$ as a full inverse subsemigroup of $T_{(U \times \vee) \times E}$. For $e \in E$,

$$
e \theta_{1} \psi_{1}=\theta_{1} \varphi_{1}^{\prime} \xi_{1}=\varepsilon_{(u, e)} \xi_{1}=\varepsilon_{((u, v), e),} \text { for any } u \in U, v \in V .
$$

similarly,

$$
e \theta_{2} \psi_{2}=\varepsilon_{((u, v), e)} .
$$

Thus $\theta_{1} \psi_{1}=\theta_{2} \psi_{2}$, as required.

\section{References}

A. H. Clifford and G. B. Preston (1961), The algebraic theory of semigroups, Math. Surveys of the American Math. Soc. 7 (Providence, R.I. 1961 (Vol. 1)).

A. H. Clifford and G. B. Preston (1967), The algebraic theory of semigroups, Math. Surveys of the American Math. Soc. 7 (Providence R.1. 1967 (Vol. 2)).

T. E. Hall (1975), 'Free products with amalgamation of inverse semigroups', J. Algebra 34, 375-385.

J. M. Howie (1964), 'The maximum idempotent separating congruence on an inverse semigroup', Proc. Edinburgh Math. Soc. (2) 14, 71-79.

J. M. Howie (1975), 'Semigroup amalgams whose cores are inverse semigroups', Quart. J. Math. Oxford (2) 26, 23-45.

W. D. Munn (1966), 'Uniform semilattices and bisimple inverse semigroups', Quart. J. Math. Oxford (2), 17, 151-159.

W. D. Munn (1970), 'Fundamental inverse semigroups', Quart. J. Math. Oxford (2), 21, 157-170.

Simon Fraser University,

Burnaby V5A 1S6, British Columbia,

Canada. 